\title{
PERCEPÇÃO DE HOMENS APÓS INFARTO AGUDO DO MIOCÁRDIO
}

\author{
Men's perception after acute myocardial infarction \\ Percepción de los hombres después del infarto agudo de miocardio
}

\section{Marina Belizário Vieira}

Pontifícia Universidade Católica de Goiás - PUC - Goiânia (Goiás) - Brasil

\author{
Wendel da Silva Souza \\ Pontifícia Universidade Católica de Goiás - PUC - Goiânia (Goiás) - Brasil
}

Patrícia Freire Cavalcante

Pontifícia Universidade Católica de Goiás - PUC - Goiânia (Goiás) - Brasil

Iracema Gonzaga Moura de Carvalho

Pontifícia Universidade Católica de Goiás - PUC - Goiânia (Goiás) - Brasil

Rogério José de Almeida

Pontifícia Universidade Católica de Goiás - PUC - Goiânia (Goiás) - Brasil

\section{RESUMO}

Objetivo: Compreender a percepção de homens sobre seus próprios sentimentos, as repercussões das mudanças no estilo de vida e a participação familiar após infarto agudo do miocárdio (IAM). Métodos: Estudo descritivo com abordagem qualitativa, realizado entre março e junho de 2016, em Goiânia, Goiás, Brasil, no qual se aplicaram entrevistas semi-estruturadas a homens adultos que tiveram episódio de infarto. A análise das entrevistas se baseou na Teoria Fundamentada nos Dados (Grounded Theory), de onde emergiram as categorias explicativas: Sentimentos vivenciados pelos pacientes após diagnóstico de IAM; Repercussão da mudança no estilo de vida com enfoque na alimentação e atividade física; Envolvimento e suporte familiar. Resultados: Em meio aos diversos sentimentos que acompanham o diagnóstico de IAM, destacaram-se o medo da finitude e de desamparar a família. As mudanças no estilo de vida, como elemento essencial do tratamento não medicamentoso, eram iniciadas. Quanto à alimentação, porém, foram insuficientes, em parte, devido à falta de orientações nutricionais adequadas. No que se refere à prática de atividade física, havia conscientização adequada, mas prevaleceu a dificuldade de manutenção do novo hábito. As famílias se revelaram a fonte majoritária de apoio social, sendo decisivas nesse processo. A adesão demonstrou-se parcial, tendo a falta de continuidade como entrave principal. Conclusão: O sucesso do planejamento terapêutico contínuo depende da adesão dos usuários, com existência de dificuldades nesse aspecto, mesmo com suporte social adequado. Torna-se clara a necessidade da conscientização sobre prevenção secundária e seus benefícios, de educação alimentar e da manutenção da mudança de hábitos.

Descritores: Estilo de Vida; Infarto do Miocárdio; Pesquisa Qualitativa; Saúde do Homem.

\section{ABSTRACT}

Objective: To understand men's perception about their feelings, repercussions of lifestyle change and family participation after acute myocardial infarction (AMI). Methods: Qualitative descriptive study conducted between March and June 2016 in Goiânia, Goiás, Brazil, using semi-structured interviews with adult men who had an episode of infarction. The analysis of the interviews was based on Grounded Theory, from which the following explanatory categories emerged: Feelings experienced by the patients after diagnosis of AMI; Repercussion of lifestyle change with focus on feeding and physical activity; Family involvement and support. Results: In the midst of the whirlwind of feelings that accompanied the diagnosis of AMI, the fear of finitude and of abandoning the family stood out. Lifestyle change, as an essential element of non-drug treatment, was initiated. However, with regard to feeding, they were partially insufficient due to the lack of adequate nutritional guidelines. As for physical activity, there was adequate awareness, but the difficulty of maintaining the new habit prevailed. Families have proved to be the main source of social support, being decisive in this process. The adherence was partial and the lack of continuity was the main obstacle. Conclusion: The success of continuous therapeutic planning depends on users'adherence, who experience difficulties in this aspect, even in the face of adequate social support. There is a clear need for raising awareness of secondary prevention and its benefits, of food education and of maintaining habit changes.

Descriptors: Lifestyle; Myocardial Infarction; Qualitative Research; Men's Health. 


\section{RESUMEN}

Objetivo: Comprender la percepción de los hombres sobre sus propios sentimientos, las repercusiones de los cambios del estilo de vida y la participación familiar después del infarto agudo de miocardio (IAM). Métodos: Estudio descriptivo de abordaje cualitativo realizado entre marzo y junio de 2016 en Goiânia, Goiás, Brasil en el cual se aplicaron entrevistas semi-estructuradas a los hombres adultos que tuvieron algún episodio de infarto. El análisis de las entrevistas se dio a través de la Teoría Fundamentada de los Datos (Grounded Theory) de lo cual emergieron las categorías explicativas: Sentimientos vividos por los pacientes después del diagnóstico de IAM; Repercusión del cambio del estilo de vida basado en la alimentación y actividad física; Envolvimiento y apoyo familiar. Resultados: Entre los diversos sentimientos que acompañan el diagnóstico de IAM se destacaron el miedo de la finitud y de abandonar a la familia. Se iniciaban los cambios del estilo de vida como elemento esencial del tratamiento sin medicación. Sin embargo, los cambios respecto la alimentación fueron insuficientes, en parte, debido la falta de orientaciones nutricionales adecuadas. Había concienciación adecuada sobre la práctica de actividad fisica pero prevaleció la dificultad para la manutención del nuevo hábito. Las familias se revelaron la principal fuente de apoyo social, siendo decisivas en el proceso. La adhesión ha sido parcial y la falta de seguimiento ha sido la principal traba. Conclusión: El éxito del planeamiento terapéutico continuo depende de la adhesión de los usuarios, con dificultades en ese aspecto, aunque con apoyo adecuado. Es evidente la necesidad de la concienciación de la prevención secundaria y sus beneficios, de la educación alimentaria y de la manutención de los cambios de hábitos. .

Descriptores: Estilo de Vida; Infarto del Miocardio; Investigación Cualitativa; Salud del Hombre.

\section{INTRODUÇÃO}

As doenças cardiovasculares (DVC) figuram como a principal causa de morte no mundo e, no Brasil, constituem a causa de $30 \%$ dos óbitos ${ }^{(1)}$. Segundo dados do Departamento de Informática do Sistema Único de Saúde (DATASUS), dos 1.210.474 óbitos que ocorreram no Brasil no ano de 2013, especificamente $28 \%$ foram atribuídos às doenças do aparelho circulatório. Além disso, as enfermidades circulatórias foram responsáveis por 10,12\% de todas as internações, culminando em um elevado gasto hospitalar ${ }^{(2)}$.

Dentre essas doenças está o infarto agudo do miocárdio (IAM), que é o termo utilizado classicamente para a área de necrose isquêmica de uma porção do músculo cardíaco causada por oclusão aguda das coronárias epicárdicas. É, em cerca de $90 \%$ das vezes, a expressão clínica da instabilização de uma placa aterosclerótica coronariana com formação de trombo obstrutivo sobrejacente ${ }^{(3)}$. Seus fatores de risco dividem-se em duas categorias: as não modificáveis, que incluem idade, sexo e hereditariedade; e as modificáveis, adquiridas com o passar dos anos e relacionadas ao estilo de vida, tais como: hipertensão arterial, tabagismo, dislipidemia, obesidade, sedentarismo, diabetes mellitus ${ }^{(4)}$.

Nos últimos anos, tem-se buscado alcançar eficácia progressivamente maior no tratamento do IAM. Para se reduzir os custos que tal tarefa traz à saúde pública ${ }^{(5)}$, que são bastante elevados ${ }^{(6)}$, há a necessidade de investimento em prevenção primária (atuar sobre fatores de risco) $)^{(7)}$ e secundária (tratar adequadamente a patologia, para reduzir a taxa de recorrência) ${ }^{(6,7)}$. A prevenção secundária se baseia na correta utilização dos medicamentos prescritos e na mudança do estilo de vida. Sua importância reside na capacidade de amenizar o risco de novos episódios de $\mathrm{IAM}^{(8)}$.

Estima-se que as DCV deverão acrescer a incapacidade ajustada para anos de vida (DALY) de 85 milhões para 150 milhões em todo o mundo até 2020, ocasionando uma baixa na produtividade global ${ }^{(1)}$.

A alteração do estilo de vida é ponto-chave na terapêutica não medicamentosa desses pacientes ${ }^{(9)}$. É considerada pelo menos tão efetiva quanto o tratamento medicamentoso ${ }^{(7)}$, e pode ser composta pela realização de atividade física regular e a modificação de hábitos alimentares. O efeito cardioprotetor advindo da prática de exercício físico regular torna-a indispensável como recurso da prevenção secundária ${ }^{(8,10)}$.

Assim, a atual pesquisa debruça-se sobre essa temática fazendo um recorte em homens, já que essa população adoece com maior frequência e é considerada mais suscetível às doenças de causa evitável ${ }^{(11)}$ quando comparada às mulheres em idade reprodutiva ${ }^{(12,13)}$. Diante do exposto, o presente estudo se faz pertinente por oferecer a oportunidade de aprofundar o conhecimento sobre as percepções de homens após IAM, o que possibilita um maior entendimento das dificuldades apresentadas, visando melhorias na qualidade do acompanhamento terapêutico e da promoção de saúde para essa população.

Nesse sentido, o foco do presente artigo situa-se na perspectiva de homens que sofreram episódio de IAM, e teve por objetivo compreender a percepção de homens sobre seus próprios sentimentos, as repercussões das mudanças no estilo de vida e a participação familiar após infarto agudo do miocárdio.

\section{MÉTODOS}

Trata-se de um estudo descritivo com abordagem qualitativa. Esse tipo de estudo envolve um conjunto de memórias, crenças, valores, ambições e atitudes, o que aborda um plano mais intimista das relações e dos fenômenos que não podem ser 
limitados ao espaço superficial da objetividade numérica ${ }^{(14)}$. Tem ainda como finalidade fornecer mais informações e dados a respeito do tema a ser pesquisado, permitindo melhor compreensão na demarcação do assunto a ser investigado ${ }^{(15)}$.

O recorte empírico estabelecido situou a coleta de dados no Serviço de Cardiologia do Hospital Escola Santa Casa de Misericórdia de Goiânia, Goiás, Brasil ${ }^{(16)}$. Os entrevistados foram selecionados a partir de prontuários do serviço de arquivamento médico e estatística (SAME) do referido hospital. Incluíram-se pacientes diagnosticados com IAM por profissional médico há mais de um ano, do sexo masculino, adulto e residente na área urbana de Goiânia, Goiás. Não participaram os usuários que não frequentavam regularmente o serviço de cardiologia e os que apresentavam déficit cognitivo.

O processo de seleção dos homens para a entrevista se deu por meio de levantamento e análise de 73 prontuários de usuários, do período de 2013 a 2015. Após essa análise foram selecionados 19 participantes que se enquadravam nos critérios estabelecidos. Todos os selecionados foram contatados via telefone para explicação dos objetivos da pesquisa e agendamento de entrevista. Desse total, oito concordaram em conceder entrevista, as quais foram realizadas pessoalmente em ambiente onde estavam presentes somente o pesquisador e o participante.

Optou-se por utilizar a entrevista semi-estruturada. Tal técnica de pesquisa social permitiu que o entrevistado respondesse, com base em suas concepções, questionamentos oriundos de um roteiro de temas previamente elaborado, mas sem que o pesquisador deixasse de atuar no processo de coleta de informações. O roteiro foi constituído por dados de identificação dos participantes (idade atual, idade no episódio de IAM, estado civil, filhos, escolaridade, atividade profissional) e por temas norteadores, para que o entrevistador não perdesse o foco do objetivo da pesquisa, orientando o fluxo de ideias do entrevistado ${ }^{(17)}$. Os temas foram: hábitos de vida antes do episódio de IAM; percepção do episódio de IAM em si; procedimentos terapêuticos pós-episódio; tratamento não medicamentoso; estratégias e dificuldades para manutenção do tratamento; envolvimento da família; impacto do tratamento não medicamentoso.

As entrevistas tiveram uma duração média de 40 minutos e foram realizadas entre os meses de março e junho de 2016. Para garantir o anonimato dos participantes, todas as entrevistas foram codificadas, sendo substituídos os nomes verdadeiros pela letra "P" referente ao termo "participante".

A análise das entrevistas se baseou na perspectiva da Teoria Fundamentada nos Dados (Grounded Theory), uma metodologia indutiva que, sem partir de teorias previamente estabelecidas, aproxima-se do objeto de estudo, permitindo a formulação de uma teoria a partir de coleta e análise sistemática de dados ${ }^{(18)}$. Empreendeu-se então a codificação dos discursos, que corresponde à análise meticulosa dos dados, comparando os achados entre os diversos elementos pesquisados, de modo a conduzir à formação de teorias explicativas sobre os fenômenos estudados com base em três etapas diferentes: codificação aberta, axial e seletiva ${ }^{(18)}$.

Na codificação aberta, os pesquisadores conceituaram, categorizaram e compararam os dados. Depois, na codificação axial, diferenciaram-se as categorias mais importantes ou centrais das subcategorias, buscando conexões entre elas. A última etapa, denominada codificação seletiva, consistiu "numa narrativa descritiva sobre o fenômeno central do estudo", sintetizando tudo que foi obtido com a coleta e codificação dos dados, explicando as diferenças e semelhanças encontradas ${ }^{(18)}$.

Após realização e análise das entrevistas, com posterior codificação dos discursos dos entrevistados, emergiram as seguintes categorias explicativas do fenômeno: Sentimentos vivenciados pelos pacientes após diagnóstico de IAM; Repercussão da mudança no estilo de vida com enfoque na alimentação e atividade física; Envolvimento e suporte familiar.

O presente estudo foi aprovado pelo Comitê de Ética em Pesquisa da Pontifícia Universidade Católica de Goiás sob o parecer $n^{\circ}$ 1.420.006, e pelo Comitê de Ética em Pesquisa do Hospital Santa Casa de Misericórdia de Goiânia sob o Parecer $n^{\circ}$ 1.460 .345 .

\section{RESULTADOS E DISCUSSÃO}

A seguir, serão apresentados os dados de identificação dos oito entrevistados. Na sequência, empreendeu-se a descrição e discussão das categorias explicativas que emergiram da codificação dos discursos dos entrevistados

\section{Dados de identificação dos entrevistados}

Os participantes são caracterizados por terem uma média de idade de 58 anos, com o episódio de IAM ocorrendo em média aos 50,5 anos. Todos estavam casados e tinham mais de um filho; além de, em sua maioria, com escolaridade de ensino fundamental incompleto e atuavam em profissões variadas.

\section{Sentimentos vivenciados após diagnóstico de IAM}

Essa categoria que emergiu dos discursos adentra na percepção dos homens sobre a doença, o medo da morte e a preocupação com os entes queridos. Observou-se, assim, que o IAM se instala de forma súbita e determina alterações difíceis e dolorosas no estado de saúde de um indivíduo que não tinha a expectativa de adoecer nem de ter a sua vida modificada em função do adoecimento. A intensa sobrecarga sensorial, imposta ao indivíduo no momento que está infartando, precipita conflitos emocionais. 
"Eu fiquei preocupado, porque eu não sabia o que era" (P4)

“Ah! Dá um desespero”. (P5)

"Eu fiquei foi muito nervoso na hora, porque eu sabia que era uma situação crítica”. (P8)

A gênese das aflições relatadas por muitos entrevistados é multifatorial e é possível catalogar as principais preocupações que permeiam a consciência deles. O medo da possibilidade de morte é expresso de forma frequente nas falas, como pode ser visto a seguir:

"Foi um desespero. Pensei que iria morrer". (P1)

"Na hora, eu tinha medo de morrer. A dor me sufocava, não sabia o que ia acontecer". (P5)

"Fiquei desesperadíssimo. Achei que a morte já viria me buscar". (P6)

A literatura já demonstrou que a doença é fonte de repulsa e sintetiza o que tende a ser o mais terrível na existência humana: a morte. Por trás de tantas angústias, existe todo um simbolismo dado ao órgão vital que é o coração, configurando-o como o mantenedor da vida. Qualquer problema que o afete é sentido como uma ameaça à vida, justificando esse medo da morte ${ }^{(19)}$.

Ainda que o instinto de conservação ou expansão da vida estivesse sendo confrontado pelo infarto, para alguns, não foi o medo da morte a fonte dos sentimentos negativos, e sim a aversão ao desamparo que a sua ausência poderia impor àqueles que ficariam, especialmente aos filhos.

"Pensei em tudo. Na familia, nos filhos. Na época, minhas filhas ainda estavam muito novas, uma com 8 e outra com 12 anos. Foi um desespero”. (P1)

"Eu estava com 38 anos, dois filhos novos, hoje minha filha tem 4 anos. Na época, meu filho tinha 9 anos. Então a gente fica desesperado, né?”. (P5)

A incerteza sobre o prognóstico, a dúvida sobre a possibilidade de tratamento e a necessidade de procedimentos terapêuticos levaram à maior ocorrência de sentimentos de ansiedade nos homens. Assim, não apenas o medo da morte, mas também o conhecimento das complicações dos testes diagnósticos, a expectativa do diagnóstico e a hospitalização contribuem para o aumento de sintomas ansiosos, medos e outras repercussões psicológicas que podem, inclusive, agravar o quadro clínico ${ }^{(20)}$.

Para alguns entrevistados, o sentimento de medo e ansiedade estava direcionado à terapêutica que seria instituída para a resolução dos sintomas excruciantes e do agravo. Para um dos participantes em especial, os conflitos emocionais residiam no desconhecimento sobre possíveis procedimentos após o infarto.

"Minha preocupação era ficar livre da dor, mas não sabia como, qual era o procedimento que iria ser feito comigo. Porque eu nunca passei por isso, nunca vi um caso semelhante. Já vi caso de gente que caiu e morreu. Mas, no meu caso, eu não sabia o que ia acontecer". (P4)

Já para outro entrevistado, ter conhecimento da estratégia de reperfusão que seria adotada não o privou dos anseios.

“Fiquei com medo, medo, medo. Ainda mais quando falaram que iriam me cortar". (P6)

Em contrapartida, alguns relatos demonstraram que o infarto agudo do miocárdio não foi um evento precipitante de abalo emocional em sua universalidade. É possível uma assimilação menos catastrófica do episódio, como se constata nas seguintes falas:

“Olha, eu nem fiquei com medo, nem abalado. Não pensei muito”. (P7)

"Mas, para mim, foi tranquilo receber o diagnóstico de IAM. Depois que eu descobri, fiquei esperando uma vaga para ser transferido do Centro de Atenção à Saúde para o hospital. Fiquei tranquilo”. (P3)

Ser portador de uma doença cardiovascular demanda uma ruptura com o cotidiano, muita das vezes com as obrigações laborais e o abandono de velhos hábitos, tudo no intuito de excluir ou controlar os fatores de risco ${ }^{(21)}$.

\section{A mudança no estilo de vida com enfoque na alimentação e atividade física}

Essa categoria explicativa descreve as dificuldades que o IAM exerce sobre a vida dos homens entrevistados. A mudança no estilo de vida é a pedra angular da prevenção secundária do IAM. A necessidade do abandono do tabagismo e do sedentarismo, da perda ponderal, da adoção de uma dieta balanceada, do tratamento da hipertensão e da diabetes mellitus e dislipidemia são sempre abordados pelo profissional de saúde que preserva as boas práticas. É nítido, todavia, que o campo das falas e das preocupações é habitado predominantemente pela necessidade de praticar exercícios físicos e de adotar uma alimentação saudável. 
No que concerne aos hábitos alimentares precedentes à doença coronariana, alguns aspectos se destacaram por sua recorrência nas falas. Um dos principais foi a ingestão de refeições copiosas, com alto teor lipídico.

"Eu me alimentava mais de carnes gordurosas, preparadas na manteiga de porco, sem regime algum. Quanto mais gordurosa a carne, melhor eu achava”. (P2)

"Comia carne de porco, aquela da manteiga grossa”. (P6)

"Gostava de carne bem gordurosa, inclusive comia a gordura da carne”. (P8)

Face ao aumento da prevalência das doenças cardiovasculares, deveria haver uma demanda cada vez maior por orientação nutricional. Esse conhecimento, ainda que não seja suficiente para a mudança de comportamento, é crucial para as pessoas adquirirem autonomia para a tomada de decisão consciente sobre sua real situação de saúde ${ }^{(21)}$. Os discursos dos entrevistados sobre as alterações dietéticas terapêuticas após IAM demonstraram certo conhecimento da necessidade de uma mudança da alimentação.

Outro ponto bastante evidente nas falas dos entrevistados foi a carência de verduras, legumes e frutas em sua dieta rotineira. Porém, uma vez mais, ao serem diagnosticados e orientados, os pacientes abandonaram o estágio de pré-contemplação, entendido como a ausência de intenção de mudar, e então alcançaram a fase da ação. $\mathrm{O}$ aspecto hegemônico encontrado nas falas foi um esforço direcionado basicamente à adoção de uma dieta hipossódica e hipolipídica.

Estudos já identificaram diversos padrões alimentares que comprovadamente reduzem o risco cardiovascular, entre os quais, citam-se: a dieta mediterrânea, a dieta paleolítica, Dietary Approaches to Stop Hypertension (DASH), a dieta vegana, a dieta de Okinawa e a do Sudoeste Asiático ${ }^{(22-24)}$. Entretanto, mais vantajoso que determinar o padrão dietético mais eficaz para prevenção e manejo das doenças cardiovasculares, é não se ater às características individuais de cada dieta, mas aos elementos compartilhados por elas ${ }^{(22)}$.

Nesse sentido, diversos aspectos poderiam ser elencados como hipóteses para as dificuldades na aceitação de mudanças na alimentação, destacando-se a disciplina necessária para alteração de hábitos já estabelecidos.

"Dificil é, pois, após estar acostumado a comer comida forte a vida inteira, fica complicado, mas isso vai sendo superado”. (P1)

“É difícil mudar o hábito, né? Tem que ter muita disciplina. Deixar o que a gente gosta é difícil”. (P5)

Em contrapartida, há indivíduos que, em momentos de necessidade crucial de mudança, exercem toda a força mental em prol do objetivo, considerando o ganho da saúde como pouco custoso a despeito da perda de hábitos de vida culturalmente apreciados.

Os itens compatíveis entre os modelos alimentares podem ser resumidos nas seguintes metas dietéticas: consumir mais frutas, vegetais, grãos integrais, fibras solúveis, legumes, carnes magras, castanhas, laticínios desnatados, soja e proteínas da soja; consumir menos frituras, carnes, carnes processadas, bebidas açucaradas, gordura trans e saturada. E, finalmente, a principal regra: preferir pequenas porções a porções copiosas ${ }^{(4,22-24)}$.

O conteúdo dos discursos dos entrevistados demonstrou que as modificações estão aquém do proposto por diversos estudos. As preocupações residem, basicamente, na redução da ingesta de alimentos fritos, do consumo de sal e do uso da gordura de porco, na preferência por carnes mais magras e aumento do consumo de frutas e verduras. Os pacientes mostraram desconhecer outros componentes importantes para uma alimentação após IAM. Assegurar que a pessoa entenda a relação entre comportamento, saúde e doença, envolvê-la em seu tratamento e educá-la sobre o padrão de dieta ideal é imperativo para o desenvolvimento de uma consciência engajada e de um indivíduo proativo ${ }^{(21)}$.

Em relação à prática de atividades físicas antes do IAM, observou-se que uma grande parte dos pacientes nunca havia praticado exercícios físicos, ou praticava atividades de forma irregular. Questionados sobre a razão desse comportamento, vários entrevistados culparam a rotina de trabalho.

\footnotetext{
"Não praticava exercícios físicos regularmente, pois o trabalho já era pesado”. (P2)

"A correria do trabalho e do dia a dia me atrapalharam”. (P7)
}

Outros, em contrapartida, reconheciam a importância da atividade física e até iniciavam a prática de alguma modalidade esportiva, porém, não eram capazes de se manterem motivados.

"Fui no cardiologista e ele me pediu para andar, mas, antes mesmo do infarto, eu parei de fazer caminhada". (P4)

O IAM, após a resolução de sua fase aguda e crítica, demanda uma atenção crônica, permanente, que se baseia em duas vertentes principais: medicamentosa e não medicamentosa. O tratamento não medicamentoso é considerado pelo menos tão efetivo quanto o uso de medicações ${ }^{(7)}$. Um dos aspectos primordiais desse tratamento é a realização de exercícios físicos regulares, que se configuram como elemento indispensável na reabilitação cardíaca após o evento isquêmico ${ }^{(25)}$. 
É interessante notar que, frente ao impactante diagnóstico de IAM, houve uma mudança de padrão comportamental, levando a maioria dos entrevistados a se engajarem ativamente na erradicação do sedentarismo.

"Faço (caminhada) todo dia, de segunda a sexta". (P2)
"Caminho em torno de 45-60 minutos todos os dias" (P1)
"Tem uns dois anos que eu tenho feito caminhada de segunda a sexta-feira". (P8)

Por outro lado, parte dos entrevistados manteve um comportamento sedentário. Nem mesmo a experiência recém-vivida com a doença coronariana foi capaz de provocar a mudança de hábitos. Alguns passaram a praticar atividade física apenas esporadicamente. Outros, por sua vez, nem mesmo iniciaram o processo de aquisição de novos hábitos.

"A caminhada me faz sentir mais leve, mais ágil. Se eu ficar dois dias sem caminhar, já sinto a diferença. As mudanças ajudam no bem-estar”. (P1)

“Senti uma melhora. A respiração é outra, o ânimo é outro. Ficava mais disposto”. (P4)

"Quando mudei os hábitos eu me senti muito bem, muito bem. Dormia melhor, ficava menos nervoso e ansioso, e mais disposto" (P5).

"Eu me sinto um pouco melhor". (P7)

As orientações recebidas durante a internação e nas consultas de acompanhamento corroboram o fato de que um programa de exercícios físicos, como forma de reabilitação cardíaca, deve ser oferecido a todo coronariopata, mostrando os benefícios do engajamento na atividade física ${ }^{26,27)}$.

Os entrevistados afirmaram que foram aconselhados a praticar caminhada, inicialmente mais leve, e então ir aumentando o ritmo e a intensidade. Estudos identificaram uma correta ênfase aos exercícios físicos após IAM $^{(28)}$, recomendando início de programa supervisionado de exercícios físicos após cerca de uma semana ${ }^{(29)}$ a dez dias da alta hospitalar, iniciando-se com 2030 minutos diários, em um nível confortável, aumentando a intensidade no decorrer da melhora do condicionamento físico e cardiovascular $^{(26,30)}$.

Não apenas aspectos subjetivos foram afetados pelas mudanças realizadas, mas também parâmetros fisiológicos objetivamente acompanhados pelos médicos responsáveis, como a pressão arterial sistêmica. Outro parâmetro essencial mencionado foi o nível sistêmico de colesterol. Finalmente, não pôde deixar de ser notada nas entrevistas a motivação central para todo o tratamento, prosseguimento e para cada mudança penosamente realizada pelos entrevistados.

Dentre os entrevistados que se devotaram à prática regular de exercícios físicos, percebeu-se que, quando questionados quanto à melhoria do bem-estar e da qualidade de vida, houve um reconhecimento dos benefícios de se exercitar. Esse achado está em conformidade com estudos que elencaram resultados positivos advindos de um programa equilibrado de atividade física. Dentre os quais podem ser citados: aumento da expectativa de vida, prevenção de eventos cardíacos, melhora da função cardíaca e do remodelamento cardíaco, redução de placas ateroscleróticas ${ }^{(28)}$; modificação dos fatores de risco cardiovascular e redução da frequência cardíaca de repouso ${ }^{(31)}$; redução da morbidade e mortalidade, com aumento da qualidade de vida ${ }^{(29)}$; diminuição da isquemia estresse-induzida e aumento da capacidade cardiovascular funcional ${ }^{(32)}$; redução de re-estenose coronariana $\operatorname{tardia}^{(33)}$; redução da progressão da doença e influência positiva sobre a função endotelial coronariana ${ }^{(34)}$; redução de internações hospitalares ${ }^{(35)}$.

\section{Envolvimento e suporte familiar}

Essa categoria explicativa tem como foco a participação familiar no processo de entendimento da doença e a coparticipação nas alterações do estilo de vida. As entrevistas demonstraram claramente a influência e o suporte exercidos pelos familiares na reeducação alimentar, assim como nas alterações ocorridas na dinâmica familiar após as orientações médicas pós-infarto.

\footnotetext{
"Todo mundo acabou mudando, e eles até se acostumaram". (P2)

"Mudou a comida da casa toda. Todo mundo procurando uma alimentação mais saudável mesmo". (P3)

"Todo mundo mudou alguma coisa e vai mudar mais agora". (P6)
}

Ao relatarem as mudanças na dinâmica alimentar familiar, todos os entrevistados, invariavelmente, declararam que apenas um tipo de alimentação era preparado em seus lares, negando a possibilidade de ser preparada uma comida à parte para os mesmos enquanto suas famílias mantinham os hábitos alimentares anteriores. Dessa forma, todos acabaram sendo afetados pelas mudanças efetuadas.

Um dos fatores que se relacionam ao cerne do tratamento não medicamentoso bem-sucedido foi o suporte familiar. Durante a fase de recuperação do IAM, os indivíduos necessitam de ajuda com sua condição psicossocial ${ }^{(36)}$. A família se torna a mais importante rede de apoio social ${ }^{(37)}$. As entrevistas demonstraram que um único tipo de alimentação era preparado, de modo que a família toda teve de se adaptar às necessidades dietéticas do paciente. Houve aceitação dos familiares, por entenderem o 
problema e se conscientizarem da importância das mudanças. Esses achados corroboram com o elevado nível de apoio por parte das famílias, prevalecendo um suporte efetivo ${ }^{(38,39)}$.

É nítido nos relatos o suporte positivo advindo do núcleo familiar, em especial da esposa, assumindo o papel de realizar as mudanças alimentares necessárias e de cobrar dos entrevistados o seguimento apropriado das orientações médicas. Para dois dos entrevistados, contudo, os membros da família que assumiram o papel de inspecionar e garantir o cumprimento das orientações médicas foram as filhas, que, em ambos os casos, são nutricionistas.

Quando questionados sobre a possibilidade de difícil aceitação da nova dieta por parte dos familiares, os entrevistados relataram que, em alguns casos, ocorreram reclamações, porém, foram resolvidas com diálogo e conscientização.

"Às vezes, meu filho até reclama dizendo que a comida não tem sal e tal, mas minha esposa fala que, desde que tive o IAM, a alimentação tinha que ser mudada por minha causa. Ele entende”. (P2)

Um dado interessante notado nas entrevistas é que a maior parte das famílias apoia, participa e cobra os entrevistados na questão alimentar. Contudo, no tocante aos exercícios físicos, poucos familiares aderiram. Houve também esposas e filhos que mudaram seus padrões de atividade física após presenciarem o sofrimento advindo da patologia do entrevistado.

A despeito de todo o suporte social e familiar, os pacientes que vivenciaram a experiência de uma patologia grave, como o IAM, frequentemente têm dificuldades na adesão ao tratamento ou em sua manutenção. Para ser efetivo, o tratamento não medicamentoso assumido após o infarto deve se tornar uma prioridade na vida dos pacientes ${ }^{(10)}$.

A importância fundamental da adesão a uma mudança do estilo de vida é percebida pela obtenção de menores taxas de doença cardiovascular e mortalidade após a adoção de alterações de hábitos não saudáveis ${ }^{(8)}$. Assim, as mudanças de estilo de vida devem ser incentivadas, a despeito das dificuldades enfrentadas para a continuidade em longo prazo, não apenas por reduzirem a recorrência de episódios de IAM, mas também por aumentarem a qualidade de vida dos pacientes ${ }^{(9,10)}$.

Estudos identificaram que os pacientes coronariopatas devidamente conscientizados tendem a buscar a adoção de uma conduta pró-ativa e também a desenvolver o autocuidado ${ }^{(10,21)}$. Por terem a vida ameaçada na fase aguda da doença, vários pacientes tomam a resolução de alterar seu estilo de vida, porém, após a estabilização do quadro, encontram dificuldades em se manter firmes, evidenciando que o grande desafio é dar continuidade ao que foi iniciado ${ }^{(40)}$.

Ainda há discordância entre diferentes literaturas quanto aos níveis de adesão ao tratamento da população em geral, na qual estudos mostram adesão de boa parte dos participantes ${ }^{(10)}$, em contraste com outros que afirmaram que a adesão dos pacientes é baixa, com um terço deles não aderindo efetivamente à prevenção ${ }^{(7)}$. Mesmo aqueles que aderem às recomendações falham em atingir as metas terapêuticas ${ }^{(41)}$.

Portanto, a grande dificuldade dos pacientes pode estar relacionada à não compreensão /aceitação de que o tratamento do IAM é crônico, a longo prazo e de que a adesão deve ser contínua. O resultado da negligência para com o tratamento proposto é que as possíveis perspectivas de futuro, para os que não observam o que é orientado, é o aumento do número de recidivas da doença, culminando em aumento da mortalidade de homens adultos ${ }^{(40)}$. Dessa forma, é mister que esses pacientes sejam sensibilizados pela equipe que os atende, para o aumento da qualidade de vida e melhoria das condições de saúde.

As entrevistas retrataram bem a percepção e vivência do agravo à saúde. Ao demonstrar os sentimentos vivenciados na experiência do IAM, a importância da alteração dos hábitos de vida e as dificuldades em praticá-las e mantê-las, a relevância imensurável do apoio familiar e os paradigmas essenciais da adesão ao tratamento, este estudo buscou contribuir na compreensão dessa patologia de grande impacto na saúde pública brasileira.

\section{CONSIDERAÇÕES FINAIS}

A confirmação de uma doença grave é fator desencadeante de sentimentos que denotam sofrimento. Ao receber o diagnóstico de IAM, o homem adulto vivencia a incerteza sobre o prognóstico, a dúvida sobre a possibilidade de tratamento, a necessidade de procedimentos terapêuticos e a perspectiva de desassistir os familiares dependentes. Entre tantos sentimentos, prevalece o medo da finitude.

Verificou-se que, após o diagnóstico recebido, houve mudanças de atitudes relacionadas à alimentação, porém ainda insuficientes como medida efetiva de controle dos fatores de risco. Os relatos corroboram a ausência de informações sobre todos os componentes alimentares que permitem dar à dieta o adjetivo salutar. Quanto à prática de exercícios físicos, as entrevistas demonstraram homens conscientes e orientados sobre a necessidade de mudança desse aspecto, mas nem sempre engajados em longo prazo na sua realização.

As complexas mudanças e os desafios advindos da experiência do IAM, do seu tratamento e acompanhamento, afetam intensamente as famílias dos pacientes. Ao mesmo tempo, demonstrou-se que o círculo familiar é a maior fonte de apoio social no enfrentamento da doença, ajudando o paciente a superar e seguir adiante, tendo influência direta e decisiva na mudança de estilo de vida. 
Todo o sucesso do planejamento terapêutico depende da adesão dos pacientes ao tratamento proposto. O presente estudo demonstrou a existência de dificuldades nesse aspecto, mesmo em face de suporte social adequado. Torna-se clara a necessidade de conscientização sobre a natureza crônica da prevenção secundária e seus benefícios, da educação alimentar e de um enfoque especial na realização e manutenção das mudanças de hábitos, o principal entrave para uma melhor qualidade de vida.

\section{REFERÊNCIAS}

1. Rocha RM, Martins WA. Manual de prevenção cardiovascular. In: Rocha RM. Epidemiologia das doenças cardiovasculares e fatores de risco. São Paulo: Planmark; Rio de Janeiro: SOCERJ; 2017. p. 10-5.

2. Ministério da Saúde (BR). Informações de saúde: estatísticas vitais, mortalidade e nascidos vivos: mortalidade geral 1996 a 2013 pela CID-10. Brasília: DATASUS; 2013.

3. Souza CF, Maehara A, Lima E, Guimarães LFC, Carvalho AC, Alves CMR, et al. Caracterização morfológica e tecidual de lesões culpadas em pacientes com infarto agudo do miocárdio com supradesnivelamento do Segmento ST após uso de fibrinolítico. Análise com ultrassom intracoronário e tecnologia iMAP®. Rev Bras Cardiol Invasiva. 2014;22(3):225-32.

4. Xavier HT, Izar MC, Faria JR Neto, Assad MH, Rocha VZ, Sposito AC, et al. V Diretriz brasileira de dislipidemias e prevenção da aterosclerose. Arq Bras Cardiol. 2013;101(4):1-20.

5. Pompa JAG, Perez JMG. Factores de riesgo para la ocurrencia de infarto agudo del miocardio en pacientes fumadores. Rev Cub Salud Pública. 2013;39(4):679-88.

6. Piegas LS, Avezum A, Guimarães HP, Muniz AJ, Reis HJL, Santos ES, et al. Comportamento da síndrome coronariana aguda: resultados de um registro brasileiro. Arq Bras Cardiol. 2013;100(6):502-10.

7. Chaves G, Britez N, Munzinger J, Uhlmann L, Gonzales G, Oviedo G, et al. Education to a healthy lifestyle improves symptoms and cardiovascular risk factors - AsuRiesgo Study. Arq Bras Cardiol. 2015;104(5):347-55.

8. Deslandes A. The biological clock keeps ticking, but exercise may turn it back. Arq. Neuropsiquiatr. 2013;71(2):113-8.

9. Soares TS, Piovesan CH, Gustavo AS, Macagnan FE, Bodanese LC, Feoli AMP. Hábitos alimentares, atividade física e escore de risco global de Framingham na síndrome metabólica. Arq Bras Cardiol. 2014;102(4):374-82.

10. Gallo AM, Laurenti R. Mudança de hábitos e atitudes em sobreviventes de infarto agudo do miocárdio e angioplastia primária. Saúde (Santa Maria). 2014:40(2):59-66.

11. Hemmi APA, Conceição JA, Santos DDM. Representações sociais de homens sobre saúde e doença: contribuições para o cuidado. Rev Enferm Cent-Oeste Min. 2015;5(1):1457-68.

12. Santos DAM, Navarro WYS, Alexandre LM, Cestari PF, Smanio PEP. Prevalência de isquemia miocárdica na cintilografia em mulheres nos períodos pré/pós-menopausa. Arq Bras Cardiol. 2013;101(6):487-94.

13. Simões RS, Sasso GRS, Silva RF, Baracat EC, Soares JM Jr.. Incidência de doenças cardiovasculares e estrogênios na pósmenopausa. Rev Bras Med. 2015;72(4):166-70.

14. Gerhardt TE, Silveira DT. Métodos de pesquisa. Porto Alegre: UFRGS; 2009.

15. Prodanov CC, Freitas EC. Metodologia do trabalho científico: métodos e técnicas da pesquisa e do trabalho científico. $2^{\text {a }}$ ed. Novo Hamburgo: Feevale; 2013.

16. Hospital Santa Casa de Misericórdia. Serviço de Cardiologia. Goiânia; 2017.

17. Manzini EJ. Entrevista semiestruturada: análise de objetivos e de roteiros. Marília: UNESP; 2004.

18. Pinto CMA Teoria fundamentada como método de pesquisa para ambientes virtuais de aprendizagem. Caminhos Linguística Aplic. 2012;7(2):78-96.

19. Sousa MZ, Oliveira VLM. Vivenciando o infarto: experiência e expectativas dos pacientes. Esc Anna Nery Rev Enferm. 2005;9(1):72-9.

20. Silva MAS, Marques PTV, Castro DFA, Padula MPC, Yano KT, Coimbra ALL, et al. Relação entre orientação, ansiedade e dor em pacientes submetidos ao cateterismo cardíaco. Arq Méd Hosp Fac Ciênc Med Santa Casa São Paulo. 2016;61(1):2834.

21. Oliveira LB, Puschel VAA. Conhecimento sobre a doença e mudança do estilo de vida em pessoas pós-infarto. Rev Eletrônica. Enferm. 2013;15(4):1026-33. 
22. Katz DL, Meller S. Can we say what diet is best for health? Annu Rev Public Health. 2014;35:83-103.

23. Martinez-Gonzales MA, Bes-Rastrollo M. Nutrition and cardiovascular disease. In: Rothkopf MM, Nusbaum MJ, Haverstick LP. Metabolic medicine and surgery. Flórida: CRC; 2014, p. 1-31.

24. Baena RC. Dieta vegetariana: riscos e benefícios. Diagn Tratamento. 2015;20(2):56-64.

25. Backshall J, Ford GA, Bawamia B, Quinn L, Trenell M, Kunadian V. Physical activity in th5 management of patients with coronary artery disease: a review. Cardiol Rev. 2015;23(1):18-25.

26. Jones K, Saxon L, Cunningham W, Adams P. Secondary prevention for patients after a myocardial infarction: summary of updated NICE guidance. BMJ. 2013;347:f6544.

27. Swift DL, Lavie CJ, Johannsen NM, Arena R, Earnest CP, O’Keef JH, et al. Physical activity, cardiorespiratory fitness, and exercise training in primar and secondary coronary prevention. Circ J. 2013;77(2):281-92.

28. Siervuli MTF, Silva AS, Silva AC, Muzzi RAL, Santos GAB. Infarto do miocárdio: alterações morfológicas e breve abordagem da influência do exercício físico. Rev Bras Cardiol. 2014;27(5):349-55.

29. Rivas-Estany E, Sixto-Fernandez S, Barrera-Sarduy J, Hernandez-Garcia S, Gonzalez-Guerra R, Stusser-Beltranena R. Efectos del entrenamiento físico de larga duración sobre la función y remodelación del ventrículo izquierdo en pacientes con infarto miocárdico de pared anterior. Arch Cardiol Méx. 2013;83(3):167-73.

30. Araújo CGS. Reabilitação cardíaca: muito além da doença coronariana. Arq Bras Cardiol. 2015;105(6):549-51.

31. Izeli NL, Santos AJ, Crescêncio JC, Gonçalves AC, Papa V, Marques F. Aerobic training after myocardial infarction: remodeling evaluated by cardiac magnetic resonance. Arq Bras Cardiol. 2016;106(4):311-8.

32. Giallauria F, Acampa W, Ricci F, Vitelli A, Maresca L, Mancini M, et al. Effects of exercise training started within 2 weeks after acute myocardial infarction on myocardial perfusion and left ventricular function: a gated SPECT imaging study. Eur J Prev Cardiol. 2012;19(6):1410-9.

33. Lee HY, Kim JH, Kim BO, Byun YS, Cho S, Goh CW, et al. Regular exercise training reduces coronary restenosis after percutaneous coronary intervention in patients with acute myocardial infarction. Int J Cardiol. 2013;167(6):2617-22.

34. Gielen S, Laughlin MH, O’Conner C, Duncker DJ. Exercise training in patients with heart disease: review of beneficial effects and clinical recommendations. Prog Cardiovasc Dis. 2015;57(4):347-55.

35. Anderson L, Oldridge N, Thompson DR, Zwisler AD, Rees K, Martin N, et al. Exercise-based cardiac rehabilitation for coronary heart disease: cochrane systematic review and meta-analysis. J Am Coll Cardiol. 2016;67(1):1-12.

36. Junehag L, Asplund K, Svedlund M. A qualitative study: perceptions of the psychosocial consequences and access to support after an acute myocardial infarction. Intensive Crit Care Nurs. 2014;30(1):22-30.

37. Garcia RP, Budó MLD, Viegas AC, Cardoso DH, Schwartz E, Muniz RM. Estrutura e vínculos de uma família após infarto agudo do miocárdio. Cuidarte. 2015;6(1):991-8.

38. Zanchet AT, Marin AH. Perfil psicossocial de pacientes com síndrome coronariana aguda. Psicol Saúde Doenças. 2014;15(3):656-70.

39. Garcia RP, Budó MLD, Schwartz E, Simón BS, Silva FM. Apoio social frente à necessidade de cuidado após infarto do miocárdio. Rev Bras Enferm. 2015;68(4):649-55.

40. Scherer C, Stumm EMF, Loro MM, Kirchner RM. O que mudou em minha vida? considerações de indivíduos que sofreram infarto agudo do miocárdio. Rev Eletrônica Enferm. 2011;13(2):296-305.

41. Pomeshkina S, Borovik IV, Barbarash OL. Adherence to non-medication treatment in patients undergoing coronary artery bypass surgery. Eur Heart J. 2013;34(1):1213-8.

\section{Endereço para correspondência:}

Marina Belizário Vieira

Pontificia Universidade Católica de Goiás - PUC

Avenida Universitária, 1440, Área 4, bloco K

Bairro: Setor Universitário

CEP: 74605-010 - Goiânia - GO - Brasil

E-mail: maribelizario@gmail.com 